Our conclusions are limited to PAS in writing dystonia however much variation exists with other plasticity protocols. Large multicentre studies of both focal and generalised forms of dystonia, probing variability of individual neurophysiological profiles are encouraged. This will reveal the true role of plasticity in the pathophysiology of dystonia and may expose subjectspecific therapeutic interventions that are currently concealed.

\title{
205 A REFLECTION ON PLASTICITY RESEARCH IN WRITING
} DYSTONIA

Anna Sadnicka, ${ }^{1}$ Masashi Hamada, ${ }^{1,2}$ Kailash Bhatia, ${ }^{1}$ John Rothwell, ${ }^{1}$

Edwards Mark'. 'National Hospital for Neurology and Neurosurgery/UCL;

${ }^{2}$ Department of Neurology, Graduate School of Medicine, The University of Tokyo

10.1136/jnnp-2014-309236.205

Much attention has focused on the hypothesis that there is enhanced plasticity of sensorimotor circuits in patients with dystonia. A common experimental method to assess plasticity in dystonia research is paired associative stimulation (PAS). Excessive, non-focal effects of PAS were observed in early studies of dystonia, however, these large effects have not been uniformly replicated.

In this viewpoint data from fifteen patients with writing dystonia is presented. We suggest that, as in healthy individuals, the effects of PAS are highly variable. A review of previous studies examining PAS in writing dystonia highlights the range of results that have been observed.

We conclude that current experimental evidence cannot be fully explained by the notion that PAS responses in writing dystonia are consistently excessive and/or non-specific. The variability of PAS responses is such that enhanced plasticity should not be considered a dystonic fingerprint as the direction of response can vary and there is overlap between patient and healthy data. We also discuss evidence questioning the assumption that PAS responses are a clear correlate to levels of synaptic plasticity; there is a need to define more specifically what PAS responses signify in the dystonic brain. 